the boundary states form a band or a discrete set of levels. In the first case one expects every captured electron to fall down to the lowest nonoccupied level. The reverse should happen during the emission process, the shallower levels being emptied first. Considering the temperature dependence of the emission rates, the longer the pulses, the more the spectrum should extend into the low-temperature region. The observed capacitance transients show no evidence for a broadening of their temperature scale. This, in addition to the observed dependence of the transient amplitude on pulse duration, is evidence for the model of the boundary states as a discrete set of levels, capable of being filled or emptied independently. ${ }^{7}$

${ }^{1}$ R. K. Mueller, J. Appl. Phys. 30, 1004 (1959).

${ }^{2}$ C. H. Seager and G. E. Pike, Appl. Phys. Lett. $\underline{35}$, 709 (1979).
${ }^{3}$ C. H. Seager, G. E. Pike, and D. S. Ginley, Phys. Rev. Lett. 43, 532 (1979).

${ }^{4}$ W. E. Taylor, N. H. Odell, and H. Y. Fan, Phys. Rev. 88,867 (1952).

${ }^{5}$ R. K. Mueller, J. Appl. Phys. 32, 635 (1961).

${ }^{6}$ G. E. Pike and C. H. Seager, J. Appl. Phys. 50, 3414 (1979).

${ }^{7}$ M. Spencer, R. Stall, L. F. Eastmann, and C. E. C. Wood, J. Appl. Phys. 50, 8006 (1979).

${ }^{8}$ A. Broniatowski, J. Phys. (Paris) 42, 741 (1981).

${ }^{9}$ R. Stratton, Proc. Phys. Soc. London, Sect. B $\underline{69}$, 513 (1956).

${ }^{10}$ The bicrystals have been provided by Cristaltec, a crystal growth division of the Centre d'Etude Nucleaire de Grenoble, France.

${ }^{11} \mathrm{~A}$. Bourret and J. Desseaux, Philos. Mag. 39, 405 (1979), Fig. 1(b).

${ }^{12}$ D. V. Lang, J. Appl. Phys. 45 , 3023 (1974).

${ }^{13}$ D. Pons, P. Mooney, and J. C. Bourgoin, J. Appl. Phys. 51, 2028 (1980).

${ }^{14}$ S. M. Sze and J. C. Irvin, Solid State Electron. 11, 599 (1968).

${ }^{15}$ A. Broniatowski, A. Blosse, P. Srivastava, and J. C. Bourgoin, to be published.

${ }^{16} \mathrm{~J}$. Bardeen, Phys. Rev. 71, 717 (1949).

\title{
Velocity Distributions of Sputtered Excited Atoms
}

\author{
Ming L. Yu, D. Grischkowsky, and A. C. Balant \\ IBM Thomas J. Watson Research Center, Yorktown Heights, New York 10598
}

(Received 19 November 1981)

\begin{abstract}
The first direct measurements are reported of the velocity distributions of sputtered atoms in excited states with electronic configurations completely different from the ground state. In contrast to previous work, the measured distributions for both the singlet and triplet metastable $D$ states of $\mathrm{Ba}$ atoms showed no energy thresholds and had most probable energies similar to those of sputtered ground-state atoms.
\end{abstract}

PACS numbers: $79.20 . \mathrm{Nc}$

Ever since the discovery ${ }^{1-3}$ of the sputtering of excited atoms during ion bombardment of solids, there have been numerous attempts to measure their velocities by monitoring the Doppler broadening $^{2,4,5}$ or the spatial distribution ${ }^{6-10}$ of the emitted light. In all these experiments the "mean" and/or "threshold" kinetic energies of the excited atoms were measured. With one possible exception, ${ }^{4}$ the sputtered atoms were found to have mean kinetic energies of hundreds of electron volts and threshold energies greater than $10 \mathrm{eV}$. Because these values are significantly higher than most probable energies of a few electron volts of the ground-state sputtered atoms and ions, they have placed strong boundary conditions on the possible mechanisms of excited-atom formation. ${ }^{11}$ Recently, Pellin, Wright, and Gruen, ${ }^{12}$ using the Doppler-shift laser fluorescence (DSLF) technique, ${ }^{13,14}$ found that the velocity distributions of sputtered $\mathrm{Zr}$ atoms in the excited $a^{3} F_{3}$ and $a^{3} F_{4}$ levels were identical to that of the ground-state $a^{3} F_{2}$ atoms. Although this result apparently contradicts the findings of all the other experiments, it was argued ${ }^{15}$ that these excited levels $(0.07$ and $0.2 \mathrm{eV}$ ) are essentially ground states because of both their low energies and the fact that they are the fine-structure components of the same electronic configuration.

In this Letter we report the first definite experimental evidence that sputtered excited atoms 
with excitation energies higher than $1 \mathrm{eV}$ and with electronic configurations completely different from that of the ground state have most probable energies comparable to those of sputtered groundstate atoms and have no kinetic energy threshold. For our experiment we used the DSLF technique to measure the velocity distributions of sputtered $\mathrm{Ba}$ atoms in the ${ }^{1} \mathrm{D}(1.4 \mathrm{eV})$ and ${ }^{3} \mathrm{D}(1.2 \mathrm{eV}) \mathrm{me}-$ tastable excited states and in the ${ }^{1} S$ ground state.

The $\mathrm{Ba}$ atom has two very favorable features for this measurement. Firstly, the lowest-energy ${ }^{1} D$ and ${ }^{3} D$ states are metastable with millisecond lifetimes. This is essential because up to $100 \mu \mathrm{sec}$ is required to traverse our observation region. Secondly, the most prevalent $\mathrm{Ba}$ isotopes have no nuclear spin. Consequently, the ${ }^{1} S$ ground state is nondegenerate, and the problem of optically pumping the ground state is eliminated.

The experimental arrangement is illustrated schematically in Fig. 1. The sample was an optically polished 1-mm-thick, 19-mm-diam, transparent $\mathrm{BaF}_{2}$ crystalline disk. A differentially pumped, mass-analyzed, ion gun provided the 1$\mu \mathrm{A}, 15-\mathrm{keV}, 1-\mathrm{mm}$-diam, $\mathrm{Ar}^{+}$beam for the sputtering at normal incidence. The chamber pressure was about $1 \times 10^{-7}$ Torr during sputtering. The tunable dye laser used was a Coherent model 599-21, pumped by a Spectra Physics 166 argonion laser. The dye-laser frequency could be tuned continuously over a $30-\mathrm{GHz}(0.34 \AA)$ range, and had a linewidth [full width at half maximum (FWHM)] of less than $3 \mathrm{MHz}$. The frequency could be set to an accuracy of $0.03 \AA$ with a Burleigh model WA20 wavemeter. The dye-laser

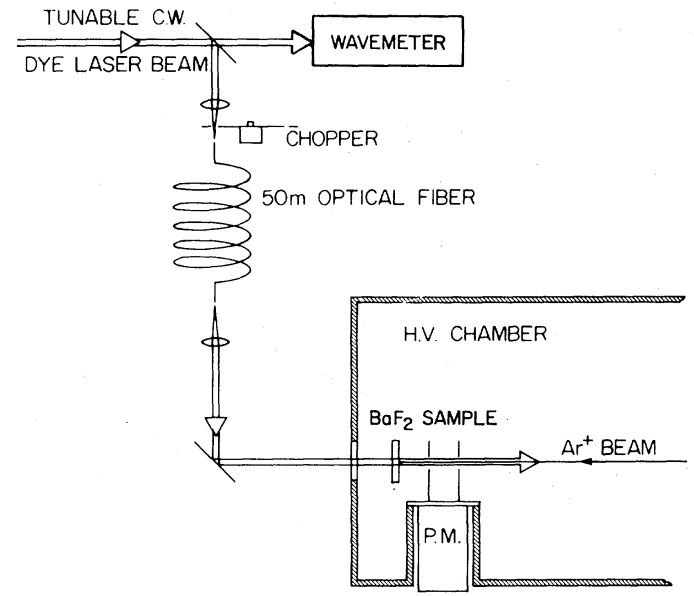

FIG. 1. Schematic diagram of the experiment. beam was focused onto a 50-m-long optical fiber, which transported the beam from the laser laboratory to the sputtering chamber. The output from the fiber was recollimated by a lens to a 1-mm-diam beam which passed through the sample and was centered on the spot bombarded by the 1-mm-diam $\mathrm{Ar}^{+}$beam on the output face. The fluorescence from the $\mathrm{Ba}$ atoms within a $25-\mathrm{mm}-$ long cylinder defined by the laser beam and starting $2 \mathrm{~mm}$ downstream from the sample was monitored at right angles by a photomultiplier. It is important to note that for all our measurements the lifetime of the monitored fluorescence was very short compared to the transit time through this cylinder.

In order to reduce optical pumping for the excited-state measurements, the dye laser was frequency modulated by passage through a lithium niobate nonlinear optical crystal to which a strong radio-frequency field at $15 \mathrm{MHz}$ was applied. This modulation produced a series of sidebands separated by $15 \mathrm{MHz}$ and extending over a range (FWHM) of approximately $240 \mathrm{MHz}$, corresponding to a velocity resolution of about $1.4 \times 10^{4} \mathrm{~cm} /$ sec. Typically, our laser powers were kept below $200 \mu \mathrm{W}$ for the excited states and below $5 \mu \mathrm{W}$ for the ground state. At these power levels we saw no variations of the measured velocity distributions with large changes in laser power, thereby indicating that optical-pumping effects were indeed negligible.

Depending on the energy level for which the velocity distribution $d N / d v$ was measured, different excitation schemes were used. As illústrated in Fig. 2, for the ${ }^{1} D$ state the Ba atoms were excited to the ${ }^{1} P$ state by laser light at 5826 $\AA$ and the uv fluorescence at $3501 \AA$ was moni-

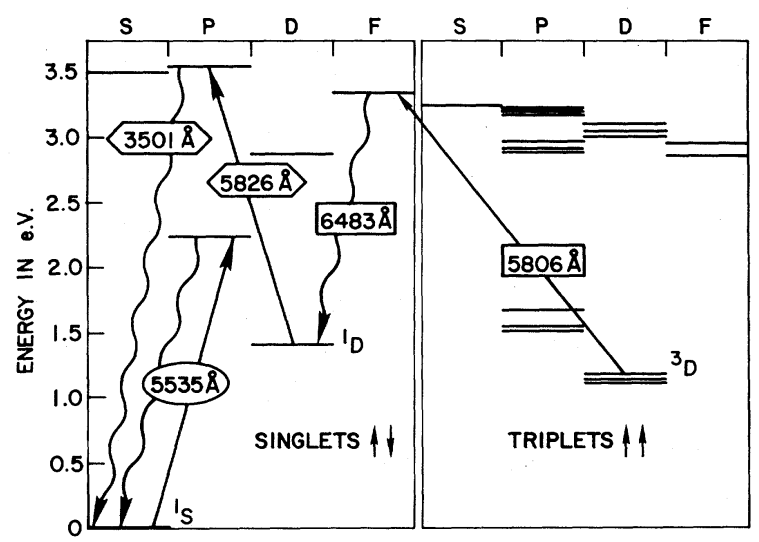

FIG. 2. Energy-level diagram for the Ba atom. 
tored by a photomultiplier shielded with glass color filters to reject the laser-light scattering. For the ${ }^{3} D$ state, the laser excitation frequency was $5806 \AA$, and the resulting fluorescence was monitored at $6483 \AA$. Again, color filters were used. In order to discriminate against the background fluorescence from the sample caused by the ion-beam bombardment, the dye-laser beam was mechanically chopped at $3 \mathrm{kHz}$ and the desired fluorescence signal resulting from the laser excitation was detected by a PAR HR-8 lock-in amplifier tuned to $3 \mathrm{kHz}$.

The measurement of $d N / d v$ for the ${ }^{1} S$ ground state required a more complex detection scheme ${ }^{14}$ to eliminate the effect of scattered laser light. For this a second lock-in amplifier was used and the ion beam was chopped at $160 \mathrm{~Hz}$. The photomultiplier output was demodulated by the first lock-in with a $Q$ of 5 at the laser chopping frequency of $3 \mathrm{kHz}$. The resulting mixer output was fed directly without filtering into the second lockin amplifier tuned to $160 \mathrm{~Hz}$ for the final stage of demodulation. For all three measurements the lock-in output signal was connected to a Tracor Northern model 1710 used as a signal averager. Typical laser-frequency scans took $100 \mathrm{sec}$ and usually fifteen or more scans were made.

The measured velocity distributions of the sputtered $\mathrm{Ba}$ atoms in the excited ${ }^{1} D$ and ${ }^{3} D$ states and the ground ${ }^{1} S$ state are shown in Fig. $3 .^{16}$ The fluorescence signals were directly proportional to the number density $d N / d v$ of sputtered atoms in the detected velocity interval. ${ }^{14,17}$ As can be seen, the linear collision cascade theory, ${ }^{18,19} \boldsymbol{d N} / \boldsymbol{d} v$ $\sim v^{2} /\left(v^{2}+v_{b}^{2}\right)^{3}$, fits $d N / d v$ of the ${ }^{1} S$ ground-state $\mathrm{Ba}$ atoms reasonably well. The solid line is the theoretical curve with $v_{b}=2.23 \times 10^{5} \mathrm{~cm} / \mathrm{sec}$, and the corresponding surface binding energy $E_{b}=3.6$ $\mathrm{eV}$ is in adequate agreement with the $E_{b}$ values of the other halides. ${ }^{20}$ The velocity distributions of both excited states are qualitatively similar. Both are significantly broader than the ground state, but the most probable velocities of $3.8 \times 10^{5} \mathrm{~cm} /$ sec for the ${ }^{1} D$ state and $3.2 \times 10^{5} \mathrm{~cm} / \mathrm{sec}$ for the ${ }^{3} \mathrm{D}$ state are quite low compared to the previous experimental work. The corresponding energies of 10.1 and $7.4 \mathrm{eV}$, respectively, remain comparable to $E_{b}$. There is no evidence of a kinetic energy threshold.

If we make the assumption that cascade transitions were relatively insignificant to our measurement, then the measured $d N^{*} / d v$ for the excited states should be the product, ${ }^{10,21} d N^{*} / d v=(d N /$ $d v) P(v) R(v)$, of $d N / d v$ for the ground-state atoms,
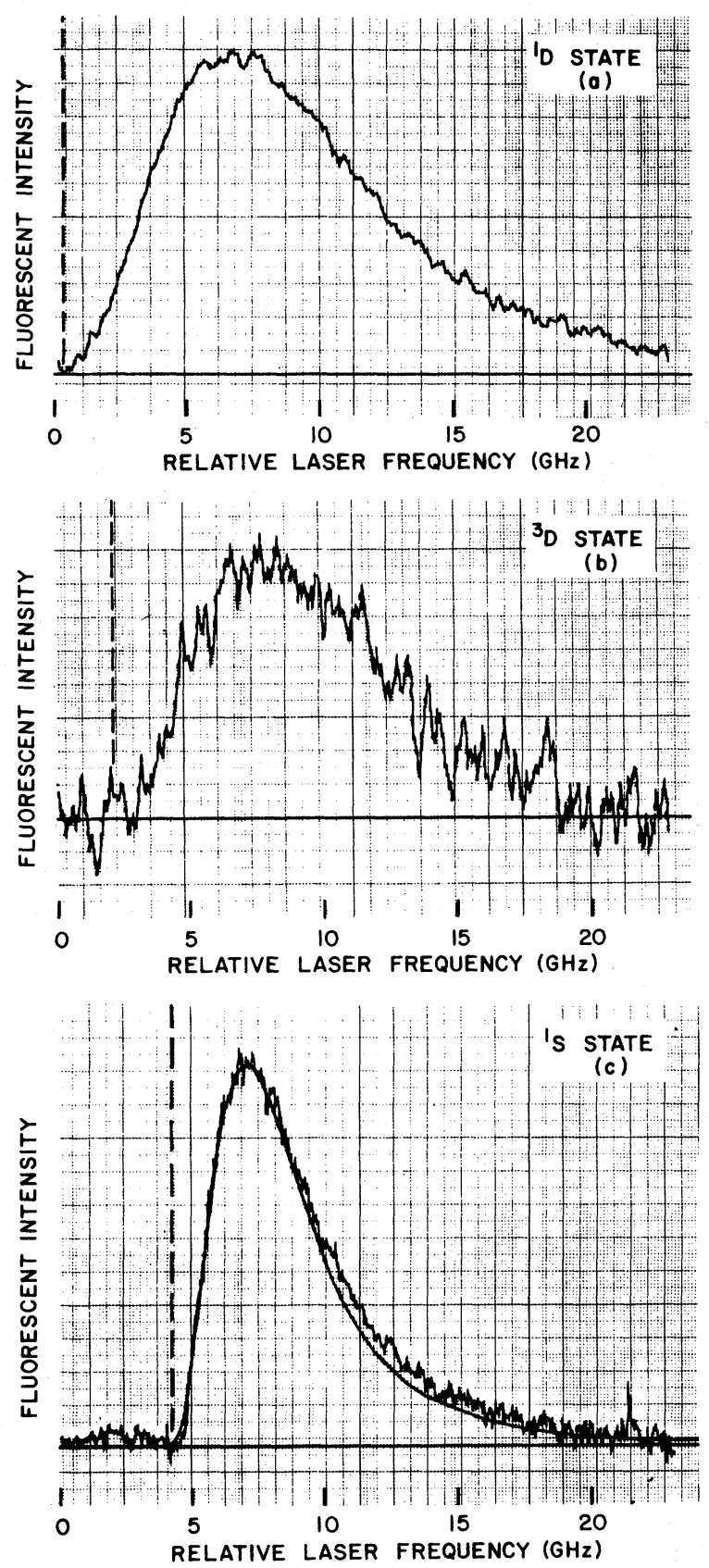

FIG. 3. Doppler-shift laser fluorescence (DSLF) measurements of $d N / d v$ for sputtered Ba atoms. Zero relative frequency indicates the beginning of the laserfrequency scans, while the dashed lines indicate the frequencies resonant with zero-velocity atoms.

(a) DSLF scan of the ${ }^{1} D(1.4 \mathrm{eV})$ excited state. A $1-\mathrm{GHz}$ Doppler shift corresponds to a velocity $v$ of $5.83 \times 10^{4}$ $\mathrm{cm} / \mathrm{sec}$ along the laser beam. The peak of the curve corresponds to the velocity $V_{p}=3.8 \times 10^{5} \mathrm{~cm} / \mathrm{sec}$ or to the kinetic energy $E_{p}=10.1 \mathrm{eV}$. (b) DSLF scan of the ${ }^{3} D(1.2 \mathrm{eV})$ excited state. $1 \mathrm{GHz}=5.81 \times 10^{4} \mathrm{~cm} / \mathrm{sec}$; $V_{p}=3.2 \times 10^{5} \mathrm{~cm} / \mathrm{sec} ; E_{p}=7.4 \mathrm{eV}$. (c) DSLF scan plus theory (solid line) of the ${ }^{1} S$ ground state. $1 \mathrm{GHz}=5.54$ $\times 10^{4} \mathrm{~cm} / \mathrm{sec} ; V_{p}=1.6 \times 10^{5} \mathrm{~cm} / \mathrm{sec} ; E_{p}=1.8 \mathrm{eV}$. 


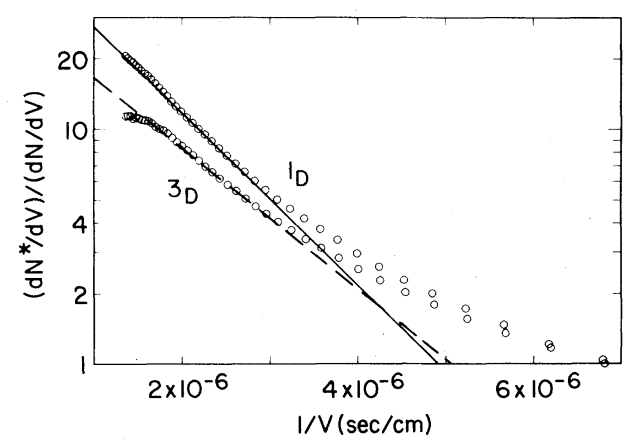

FIG. 4. The unnormalized ratio (circles) of smoothed curves from Fig. 3 for the excited-state velocity distribution $d N^{*} / d v$ to the ground-state distribution $d N / d v$ vs the reciprocal of the velocity.

the probability $P(v)$ of a sputtered atom with velocity $v$ being in the excited state, and the probability $R(v)$ of the excited state surviving nonradiative deexcitation. It is often proposed that $\boldsymbol{P}(v)$ has a weak $v$ dependence and that $R(v)$ is given by $\exp (-A / a v)$, where $A$ is a transition rate and $1 / a$ is a characteristic distance of the atom-surface interaction. ${ }^{2,3,10,21}$ Recently, $\mathrm{N} \phi$ rskov and Lundqvist ${ }^{22}$ also showed theoretically that the product $P(v) K(v)$ for the ionization of sputtered atoms has a similar exponential dependence on the velocity, although the velocity dependences of $P$ and $R$ are not separable. As shown in Fig. 4, the ratio, $\left(d N^{*} / d v\right) /(d N / d v)$ $=P(v) R(v)$, approaches an exponential dependence on $1 / v$ for both excited-state distributions. The deviation at low velocities is not unexpected in view of the predicted ${ }^{23}$ and observed ${ }^{24}$ velocity dependence of $P(v)$ for secondary ions. The $A / a$ values obtained from the indicated straight lines are $A / \boldsymbol{a}=0.9 \times 10^{6}$ and $0.7 \times 10^{6} \mathrm{~cm} / \mathrm{sec}$ for the ${ }^{1} D$ and ${ }^{3} D$ states.

We would like to acknowledge many stimulating and informative discussions with Roger Kelly. Helpful comments on the manuscript were provided by Roger Kelly and W. Reuter. Time on the ion beam machine was generously provided by W. Reuter. This work was partially supported by the U. S. Office of Naval Research.

${ }^{1}$ H. Sporn, Z. Phys. 112, 278 (1939); C. Snoek, W. F. van der Weg, and P. K. Rol, Physica (Utrecht) 30, 341 (1964)。For a historical survey, see G. E. Thomas, Surf. Sci. 90, 381 (1979).

${ }^{2}$ W. F. van der Weg and D. J. Bierman, Physica (Utrecht) 44, 206 (1969)。

${ }^{3}$ C. W. White and N. H. Tolk, Phys。Rev. Lett. 26, 486 (1971).

${ }^{4}$ C. W. White, D. L. Sims, N. H. Tolk, and D. V. McCaughan, Surf. Sci. 49, 657 (1975).

${ }^{5}$ C. M. Loxton, R. J. MacDonald, and P. J. Martin, Surf. Sci. 93, 84 (1980); C. M. Loxton, R. J. MacDonald, and E. Taglauer, Surf. Sci. 102, L76 (1981).

${ }^{6}$ T. S. Kijan, V. V. Gritsyna, and Ya. M. Fogel, Nucl. Instrum. Methods 132, 435 (1976), and references cited therein.

${ }^{7}$ H. Kerkow, Phys. Status Solidi (a) 10, 501 (1972).

${ }^{8}$ M. Braun, B. Emmoth, and I. Martinson, Phys. Scr. 10, 133 (1974).

${ }^{9}$ I. S. T. Tsong and N. A. Yusuf, Nucl. Instrum. Methods 170,357 (1980).

${ }^{10}$ S. Dzioba, O. Auciello, and R. Kelly, Rad. Eff. 45 , 235 (1980); S. Dzioba and R. Kelly, Surf. Sci. 100, 119 (1980).

${ }^{11} \mathrm{R}$. Kelly, in Inelastic Particle-Surface Collisions, edited by E. Taglauer and W. Heiland, Springer Series in Chemical Physics, Vol. 17 (Springer, New York, 1980).

${ }^{12}$ M. J. Pellin, R. B. Wright, and D. M. Gruen, J. Chem. Phys. 74, 6448 (1981).

${ }^{13}$ D. Hammer, E. Benes, P. Blum, and W. Husinsky, Rev. Sci. Instrum. 47, 1178 (1976).

${ }^{14}$ M. L. Yu, D. Grischkowsky, and A. C. Balant, Appl. Phys. Lett. 39, 703 (1981).

${ }^{15} \mathrm{R}$. Kelly, private communication.

${ }^{16}$ To check the zero-velocity point, the laser beam was reflected back by a small mirror at the far end of the vacuum chamber so that $d N / d v$ was also measured with the laser beam going against the sputtered-atom flux. The result was a diminished mirror image of $d N / d v$ about the dashed $v=0$ line in addition to the $d N / d v$.

${ }^{17}$ R. B. Wright, M. J. Pellin, D. M. Gruen, and C. E. Young, Nucl. Instrum. Methods 170, 295 (1980).

${ }^{18}$ M. W. Thompson, Philos. Mag. 18, 377 (1968).

${ }^{19}$ P. Sigmund, Phys. Rev。 184, 383 (1969).

${ }^{20} \mathrm{M}$. Szymonski, H. Overeijnder, and A. E. De Vries, Rad. Eff. 36, 189 (1978).

${ }^{21}$ R. B. Wright and D. M. Gruen, J. Chem. Phys. $\underline{72}$, 147 (1980).

${ }^{22}$ J. K. Nørskov and B. I. Lundqvist, Phys. Rev. B 19 , 5661 (1979).

${ }^{23}$ Z. Sroubek, K. Zdansky, and J. Zavadil, Phys. Rev。 Lett. $\underline{45}, 580$ (1980).

${ }^{24}$ Ming L. Yu, Phys. Rev. Lett. 47, 1325 (1981)。 